\title{
Implementation of a reaction-diffusion process in the Abaqus finite element software
}

\author{
Elisabeth Vasikaran ${ }^{1}$, Yann Charles ${ }^{1, *}$, and Pierre Gilormini ${ }^{2}$ \\ ${ }^{1}$ LSPM, Université Paris 13, Sorbonne Paris Cité, CNRS UPR 3407, 99 av. Jean-Baptiste Clément, 93430 Villetaneuse, France \\ ${ }^{2}$ Laboratoire PIMM, ENSAM, CNRS, CNAM, 151 Bd de l'Hôpital, 75013, Paris, France
}

Received: 7 October 2019 / Accepted: 27 January 2020

\begin{abstract}
To increase the Abaqus software capabilities, we propose a strategy to force the software to activate hidden degrees of freedom and to include extra coupled phenomena. As an illustration, we apply this approach to the simulation of a reaction diffusion process, the Gray-Scott model, which exhibits very complex patterns. Several setups have been considered and compared with available results to analyze the abilities of our strategy and to allow the inclusion of complex phenomena in Abaqus.
\end{abstract}

Keywords: Reaction-diffusion / finite elements / user subroutines / Gray-Scott model

\section{Introduction}

Simulating the effect of impurities on the integrity of structures leads to account for several interactions between, e.g., the mechanical fields, the impurities transport and trapping, the thermal fields, etc. The simulation of all these phenomena simultaneously is a complex task, especially when strong couplings are involved or investigated: in the hydrogen embrittlement of metals [1], or in the hydrolysis of polymers $[2,3]$, for instance, mobile species are adsorbed, transported through the material, and trapped on specific sites whose density is time and space dependent [4,5] (e.g., through the development of plasticity for hydrogen in metals [6]). Furthermore, mechanical fields can be affected by these species because of induced deformations or through modifications of mechanical properties $[7,8]$.

Numerous studies account for such interactions in finite element codes, in various application fields (metal/ hydrogen, water/polymer, metal/lithium ions, see [9-17] among others), but very few developments include several phenomena in the computations $[18,19]$, especially in the commercial finite element codes, due to their inherent limitations in terms of available degrees of freedoms at each node. Such an inclusion may, however, be of importance, e.g., to model the behavior of structures in the presence of both impurities and evolving thermal boundary conditions [20]. The aim of this work is thus to introduce some developments performed in Abaqus to solve coupled mechanical-multidiffusion finite element problems. This paper is limited to a reaction-diffusion process between two

\footnotetext{
* e-mail: yann.charles@univ-paris13.fr
}

species, which is solved by using a coupled mechanicaldiffusion scheme ('coupled temp-displacement' in Abaqus) that allows further developments to account for the mechanical fields as well. First, the multidiffusion implementation strategy is presented, and then an application to the Gray-Scott reaction-diffusion model is presented to illustrate the new capabilities $[21,22]$. We would like to underline that the proposed strategy allows an increase of the multiphysics simulations in Abaqus (including strong couplings), while keeping the native features of the software in term of modeling library (including mechanical behaviors). The application is here limited to a classical problem in order to illustrate such possibilities, but extensions and applications are widely open to a lot of coupled problems that include mechanics (metal/hydrogen, water/polymer, metal/lithium ions as previously underlined, but also electro-magneto-mechanics, etc.).

\section{Introduction of a multidiffusion process in Abaqus}

In order to solve a complex problem with mechanics and multidiffusive fields in a finite element (FE) software, it is mandatory (i) to have a finite element formulation that includes as many degrees of freedom (DOFs) per node as the number of unknown fields, and (ii) to introduce the correct weak formulation for all of these DOFs for solving the problem. Introducing extra DOFs is complex; one may exploit the unused mechanical DOFs (rotations, numbered from 3 to 6 , or the third displacement component in $2 \mathrm{D}$ problems), adding extra features to the elements (see $[23,24]$ for phase field implementation in Abaqus) 


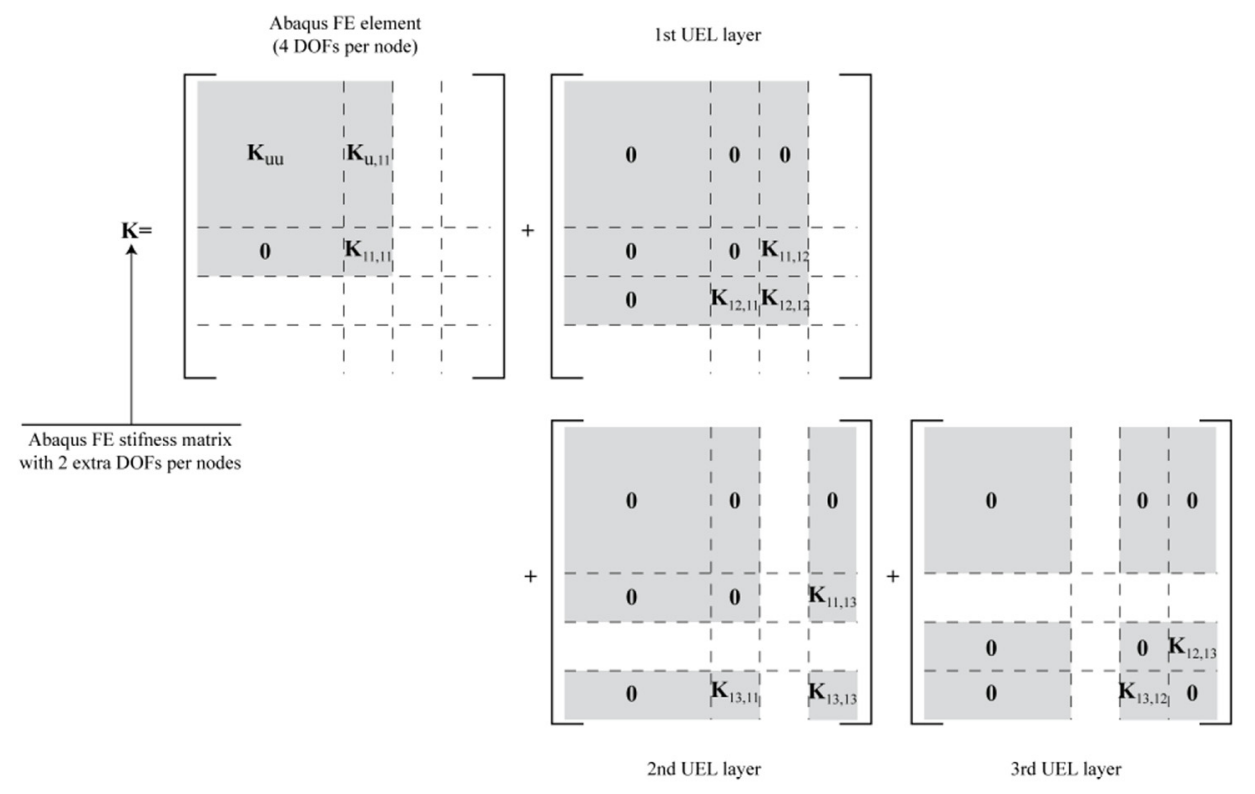

through an User ELement (UEL) routine [25]. One approach of particular interest has been proposed by Chester [26] to solve coupled thermo-chemo-mechanical problems in polymers (this work has been applied in [27] for a simple adsorption process). In this work, an UEL has been developed that activated extra DOFs, in addition to the introduction of a relevant weak formulation as specified in [25]. Such DOFs are included by default in the Abaqus element library for 'coupled temp-displacement' procedures, but they are hidden and cannot be accessed through the CAE interface or input files ${ }^{1}$. For the 'coupled tempdisplacement' procedure', there are 7 DOFs at each node of a 3D mesh, numbered as follows by Abaqus:

- 1 to 3 correspond to displacement;

- 4 to 6 correspond to rotation;

- 11 corresponds to nodal temperature (NT11 in the "Field Output" section of the CAE).

The extra DOFs, activated using an UEL, are numbered from 12 to 30, corresponding to NT12 to NT30 (for 'Nodal Temperature') variables. Once activated, their boundary conditions can be imposed in the input file and their values (NT12 $\rightarrow 30$, HFL12 $\rightarrow 30$, etc.) can be required in the output database file.

It is worth noting that all the studies mentioned above, in which an UEL was used to redefine the problem, have also superimposed an additional layer of element taken from the Abaqus library in order to visualize the results. As demonstrated in [29], it is possible to go further and extend the Abaqus finite element formulation by superimposing an UEL to an Abaqus element: the terms that are not included by default in the formulation can be introduced through the UEL. The approach chosen in the present study combines the advantages of keeping the features of the Abaqus libraries (materials, elements, etc.) and of adding extra terms and DOFs in the finite element formulation by using a superimposed UEL. Thus, the implementation work is optimized

\footnotetext{
${ }^{1}$ Their presence can be inferred from [28], sections 28.3.6 and 28.6.5, in the 'Output' subsection.
}

because the mechanical behavior does not need to be redefined. Even if a multidiffusion process only is considered here, the ultimate goal of a fully coupled mechanicalmultidiffusion problem has been kept in mind during the developments.

\section{Implementation process}

Our strategy is presented in Figure 1: several element layers sharing the same nodes are defined, and a 'coupled temp-displacement' procedure is used. In this example, the three UEL layers have the same numbers of DOFs and, assuming that DOFs 11,12 , and 13 represent diffusion DOFs between which a reaction may occur, all user elements layers share the same UEL routine with different parameters. Each layer, in this example, has a specific role:

- Layer 1: the Abaqus element (with mechanical DOFs 1 to 6 , and 11) involves the mechanical behavior, one diffusion phenomenon (related to DOF 11), and its effects on the mechanical behavior. The problem is strongly coupled (i.e., the diffusion and the mechanical problems are solved simultaneously), but no effect of mechanical DOFs on diffusion is possible here (except with developments beyond the scope of this work).

- Layer 2: this UEL layer activates DOF 12 and its coupling with the standard 'coupled temp-displacement' DOFs $1 \rightarrow 6$ and 11 , through its weak formulation.

- Layer 3 has the same role as layer 2, but for DOF 13.

- Layer 4 defines only the relation between DOFs 12 and 13.

It is worth noting that other approaches can be considered in the superimposition process (for instance, a single UEL can be used to activate DOFs 12 and 13, and to introduce all the ingredients needed in Abaqus). Each element layer leads to the computation of a specific stiffness matrix, performed either by Abaqus or by the UEL, as shown below:

see equation (1) above. 


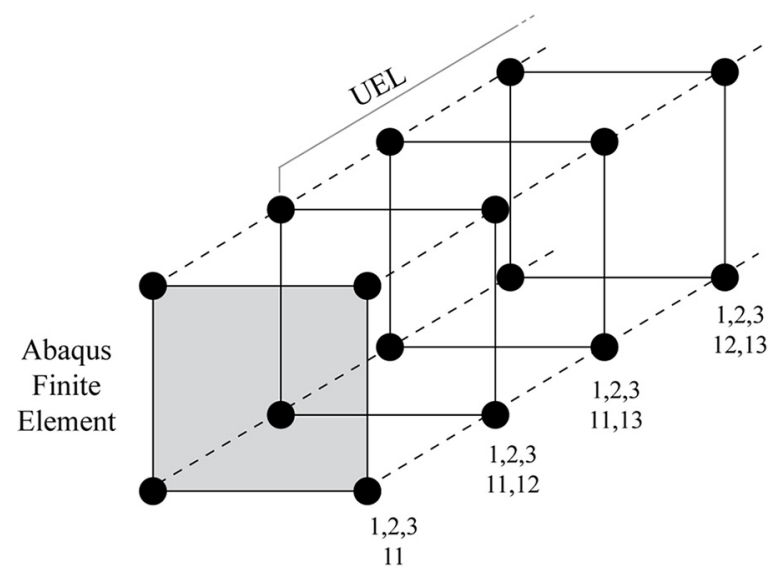

Fig. 1. Principle of the implementation of a multidiffusion process.

In the case presented in Figure 1, the stiffness matrices are $16 \times 16$ and $20 \times 20$ for the Abaqus element and for the UEL, respectively. At the end of the superimposition process, the stiffness matrix of the global problem is $24 \times 24$ : due to the activation of the extra nodes, the initial Abaqus element stiffness matrix has increased significantly, without any other user manipulation than the activation of hidden DOFs.

This strategy is applied below, where only DOFs 12 and 13 are considered, for illustration. The transient 'coupled temp-displacement' procedure is used, even if there is no coupling between DOFs $(12,13)$ and $(1,2,3,11)$ in the present work.

\section{Application}

The Gray-Scott model is considered here as a test reactiondiffusion process to be implemented.

\subsection{The Gray-Scott model}

The Gray-Scott (GS) reaction-diffusion model represents a particular case of Turing systems [30], where the reactions of three chemical species are focused on. These species, $U, V$ and $P$, define an autocatalytic system so that $[21,22]$

$$
\left\{\begin{array}{l}
U+2 V \rightarrow 3 V \\
V \rightarrow P
\end{array}\right.
$$

The space-time evolution of species $U$ and $V$ can be obtained by solving the following system of differential equations:

$$
\left\{\begin{array}{l}
\frac{\partial u}{\partial t}=D_{u} \Delta u-u v^{2}+F(1-u) \\
\frac{\partial v}{\partial t}=D_{v} \Delta v+u v^{2}-(F+k) v
\end{array}\right.
$$

where $u$ and $v$ denote the concentrations of species $U$ and $V$, respectively, $D u$ and $D v$ represent their diffusion coefficients, $F$ is the feed rate for $U$ and $k$ the kill rate for $V$. This reaction has been widely studied as a simple model to reproduce the patterns observed in several chemical reactions (or natural ones [31]), as illustrated in Figure 2.

\subsection{Numerical implementation}

The patterns induced by the GS model have been the subject of numerous studies from the seminal work by Pearson [36] (see, e.g., [37-42]), including many for entertainment purposes ${ }^{2}$, and a classification of the GS patterns has been proposed (see Fig. 3), depending on the $(F, k)$ values. Consequently, many implementations of the GS reaction can be found, based on finite differences and forward Euler integration scheme for efficiency reasons ([43-45], among others, and the very complete webpage of R. Munafo [46]), mainly in 2D. Very few [47-49] apply the finite element method, especially Abaqus. One study [50] includes mechanical coupling, but no indication on the implementation process is given, nor if extra DOFs have been introduced, unfortunately.

We have implemented the GS reaction in Abaqus by introducing DOFs 12 and 13; the details of the RHS vector and of the AMATRX matrix have been adapted from [48] by considering constant diffusion coefficients, in particular. Computations have been performed with the 'coupled temp-displacement' procedure, even if no mechanical nor temperature field is computed. It is especially worth noting that, in addition to the GS reaction simulation presented here, a full "coupled-temp displacement" Abaqus simulation can be run in the same computation. In order to evaluate the ability of our implementation to simulate a GS process accurately, all the results are compared with those given by the Python script written by D. Bennewies [44].

\subsection{Configuration studied}

The configuration studied is a square domain $2.5 \times 2.5 \mathrm{~mm}^{2}$, which is meshed with $250 \times 250$ fully integrated linear square elements (i.e., with an element size of $0.01 \times 0.01 \mathrm{~mm}^{2}$ ), over which an user element is superimposed, sharing the same nodes, for the activation of DOFs 12 and 13 (representing the concentrations of $U$ and $V$, respectively) and for the integration of the reaction-diffusion process. A transient 'coupled tempdisplacement' procedure is applied. Periodic boundary conditions are prescribed to DOFs 12 and 13 along the border of the domain, as set in [44]. The following initial conditions for $u$ and $v$ are defined using a DISP user subroutine:

$$
\left\{\begin{array}{l}
x \in \Omega \Longrightarrow u=0.5-0.01 \delta(x) ; x \notin \Omega \Longrightarrow u=1 \\
x \in \Omega \Longrightarrow v=0.25+0.01 \delta(x) ; x \notin \Omega \Longrightarrow v=0
\end{array}\right.
$$

\footnotetext{
$\overline{2}$ For instance, 'Gray-Scott reaction diffusion' keywords in YouTube gives 779 results.
} 


\section{CIMA (chlorite-iodide-}

malonic acid) reaction in

various experimental

conditions [32].

FIS (ferrocyanide-iodate-

sulfite) reaction in various

experimental conditions

[33].

\author{
CDIMA (chlorine dioxide, \\ iodine, malonic acid) \\ reaction after $12,20,35$ and \\ 52 minutes [34].
}

Belousov-Zhabotinsky

(BZ) reaction [35]
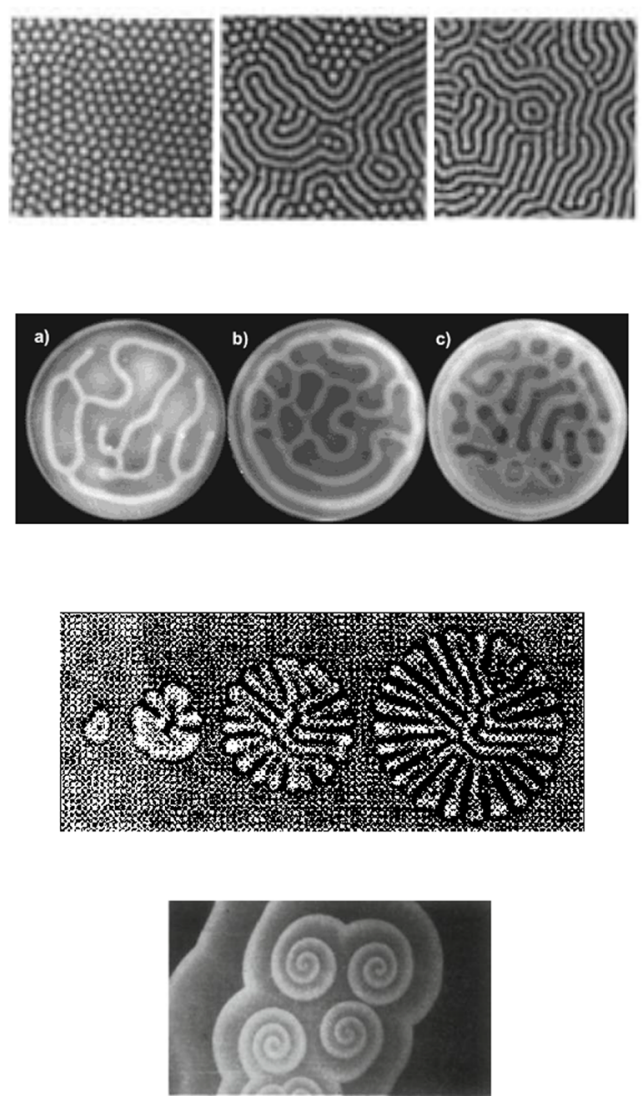

Fig. 2. Examples of chemical patterns.

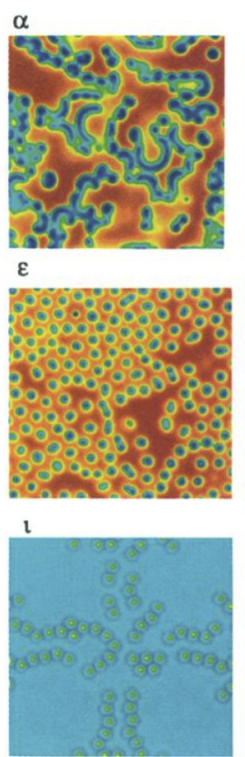

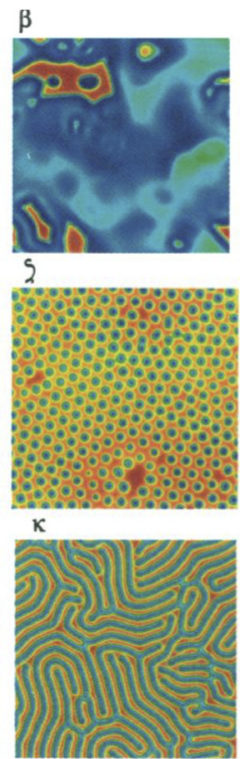

(a)

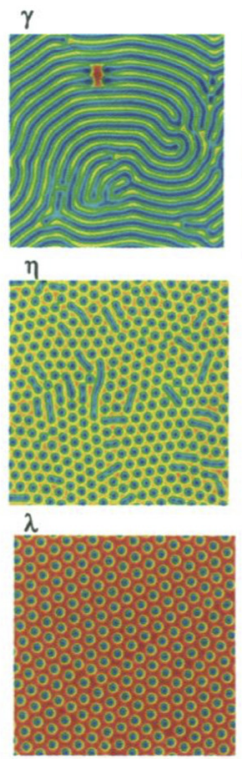

(a)

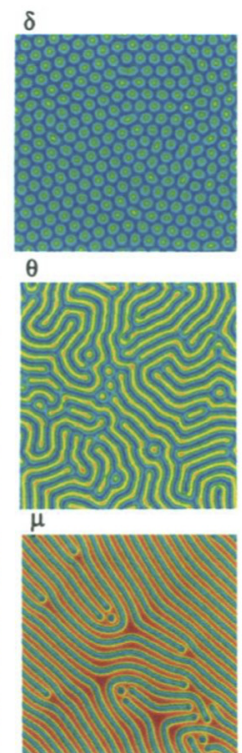

Fig. 3. (a) Types of patterns obtained with the GS reaction, and (b) their position in the $(F, k)$ plane (using $D_{u}=D_{v}=2 \times$ $\left.10^{-5} \mathrm{~mm}^{2} \mathrm{~s}^{-1}\right)$ as defined in [36]. For $(F, k)$ points where no pattern is specified, a constant homogeneous field for $u$ as well as for $v$ is expected (denoted as $B$ and $R$ for "Blue" $-u \approx 0.3$ and $v \approx 0.25$ - and "Red" $-u=1$ and $v=0$ - respectively).

where $\Omega$ is a rectangular domain $0.125(1+\delta) \times$ $0.125(1+\delta)$ with $\delta \in[01]$ a random perturbation Finally, $D_{u}$ and $D_{v}$ have been set to $10^{-5} \mathrm{~mm}^{2} / \mathrm{s}$ Table 1.

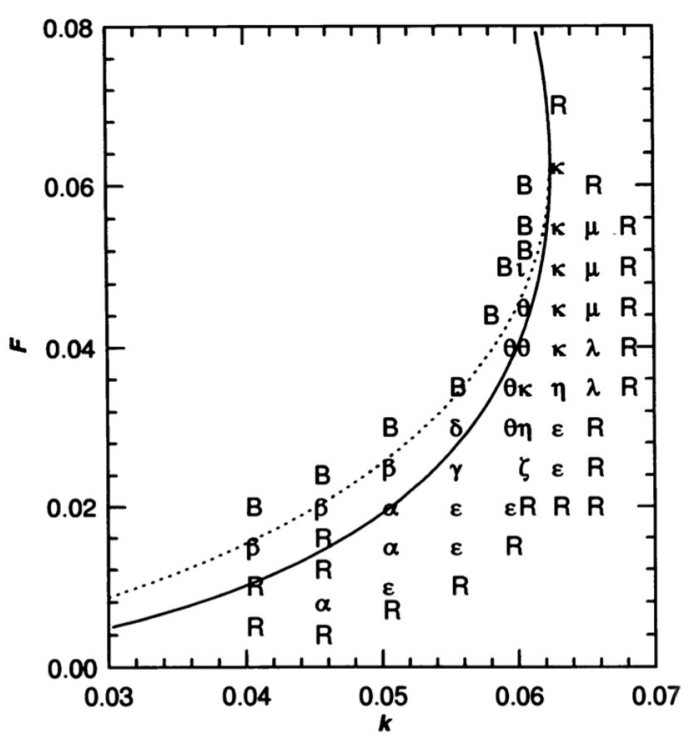

(b) 
Table 1. Reaction parameters considered (among those of [44]).

\begin{tabular}{llllll}
\hline$F$ & 0.006 & 0.022 & 0.026 & 0.046 & 0.062 \\
$k$ & 0.037 & 0.049 & 0.061 & 0.063 & 0.0609 \\
$\begin{array}{l}\text { Expected pattern } \\
{[44,46]}\end{array}$ & $\begin{array}{l}\text { Propagating } \\
\text { wavefronts }(\text { Type } \xi)\end{array}$ & $\begin{array}{l}\text { Chaotic oscillations } \\
(\text { Type } \beta)\end{array}$ & $\begin{array}{l}\text { Solitons } \\
(\text { Type } \lambda)\end{array}$ & $\begin{array}{l}\text { Worms } \\
(\text { Type } \mu)\end{array}$ & $\begin{array}{l}\text { Negatons } \\
(\text { Type } \pi \text { ) }\end{array}$ \\
\hline
\end{tabular}
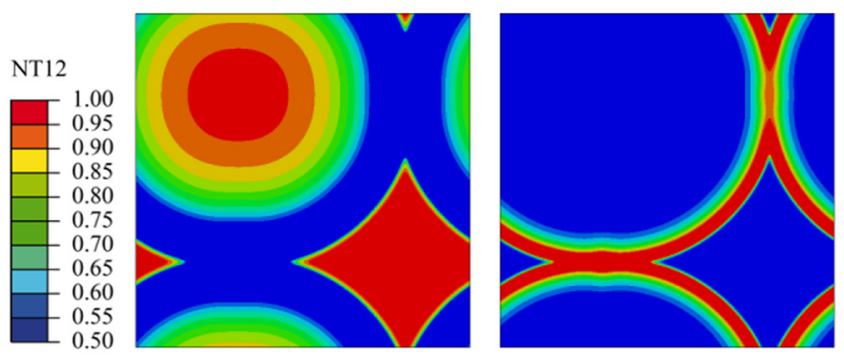

(a)

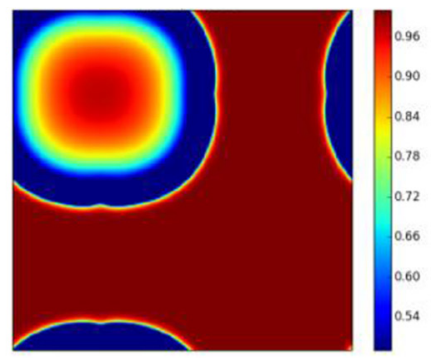

(b)

Fig. 4. (a) $u$ and $v$ fields obtained with Abaqus and (b) $u$ field computed with python following $[44]$, using $(F, k)=(0.006,0.037)$ at $t=800 \mathrm{~s}$.
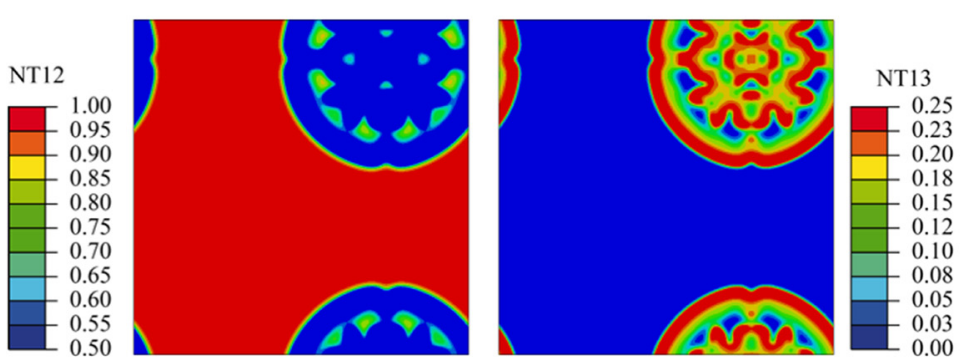

(a)

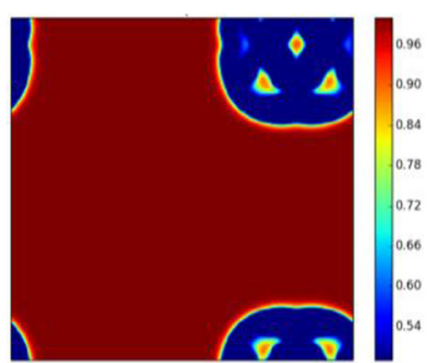

(b)

Fig. 5. Same as Figure 4 , with $(F, k)=(0.022,0.049)$ at $t=800 \mathrm{~s}$.

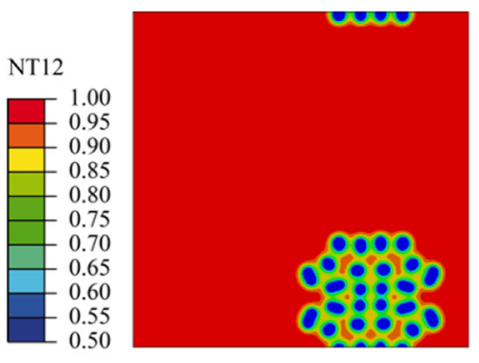

(a)

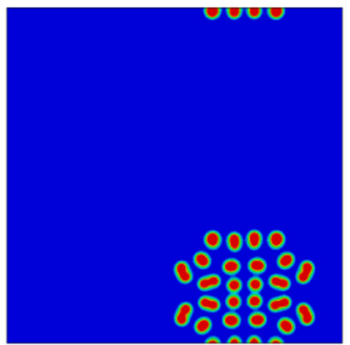

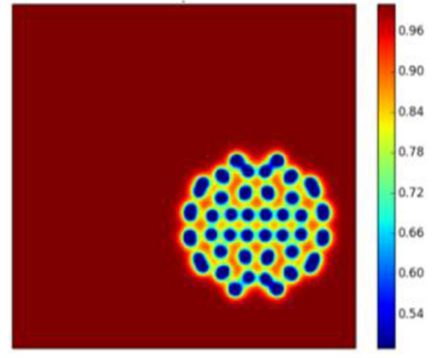

(b)

Fig. 6. Same as Figure 4 , with $(F, k)=(0.026,0.061)$ at $t=2500 \mathrm{~s}$.

\subsection{Results}

The Abaqus results for $\mathrm{u}$ (NT12) and $\mathrm{v}$ (NT13) are presented in Figures $4 \mathrm{a}-8 \mathrm{a}$, with the corresponding Python reference results for $u$ shown in Figure $4 \mathrm{~b}$. All Abaqus computations have been performed with a constant time increment of $10 \mathrm{~s}$, while the python's one is equal to $1 \mathrm{~s}$. It can be observed that our implementation in the Abaqus code is able to reproduce quite well the results obtained with another software, for various configurations. 

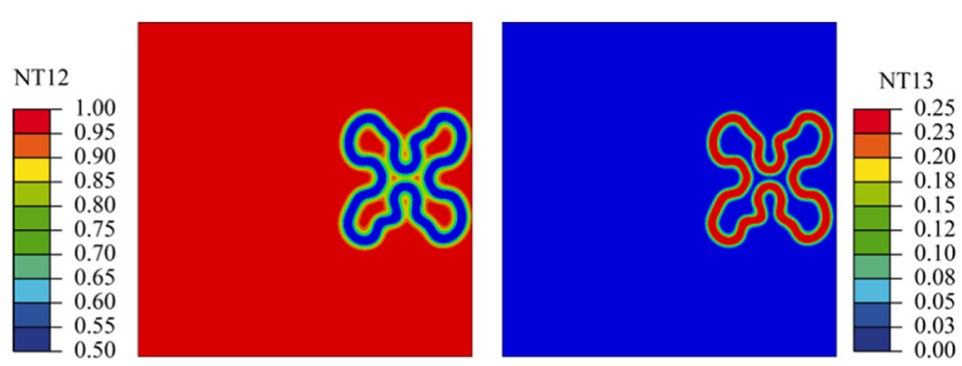

(a)

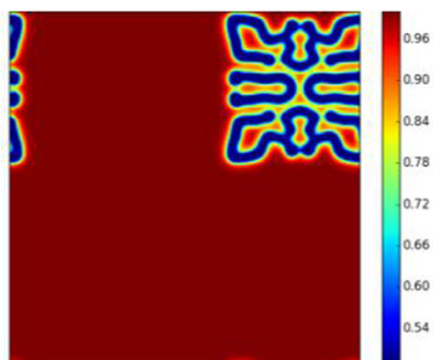

(b)

Fig. 7. Same as Figure 4 , with $(F, k)=(0.046,0.063)$ at $t=5000 \mathrm{~s}$.
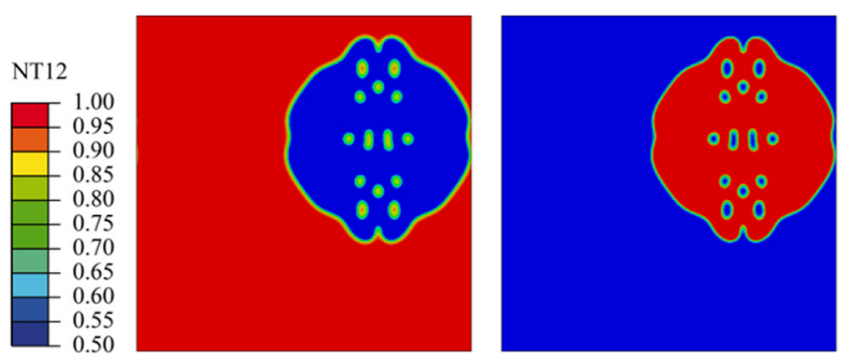

(a)

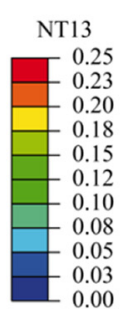

0.00

Fig. 8. Same as Figure 4 , with $(F, k)=(0.062,0.0609)$ at $t=5000 \mathrm{~s}$.

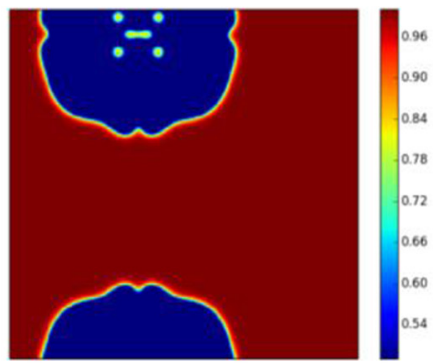

(b)
It may be noted that the U-skate geometries exhibited by Munafo [41,46]) could not be generated, as in [44].

\section{Discussion}

An important feature observed in our simulations is a non constant velocity of the pattern front, with a strong influence of the $(F, k)$ parameters. This behavior is consistent with results obtained by other methods, especially in [44]. From the Figures 4 to 8 , it might be observed that the front velocity computed by Abaqus has the same order of magnitude than the one obtained using Python.

We have also investigated the effects of the element size and of the time increment (see [46] for a more complete investigation of the time increment influence). The influence of the element size is illustrated in Figure 9 for $(F, k)=(0.006,0.037)$. When the element size increases, the generated pattern is strongly influenced by the mesh structure and tends to a square rather than a circle. Moreover, the velocity of the pattern front is increased because of a rapidly vanishing $V$ field that annihilates the reaction process.

In contrast, decreasing the time increment has no influence on the Abaqus results and on their consistency with [44], except for $(F, k)=(0.022,0.049)$ where the intensities of the pattern oscillations decrease and a steady state is finally reached for $t$ about $3400 \mathrm{~s}$.
For this configuration, the influence of the time increment is shown in Figure 10 when it is decreased from $10 \mathrm{~s}$ to $1 \mathrm{~s}$, no steady state is reached with Abaqus up to $5000 \mathrm{~s}$ and chaotic oscillations are observed, as in [44].

\section{Conclusion}

An appropriate application of user elements allows the extension of Abaqus capabilities, including the modification of library elements, the activation of hidden DOFs, and the addition of various physical processes with or without couplings. This study has been focused on the activation of DOFs and on the addition of chemical reactions in Abaqus. An application to the Gray-Scott model has been made successfully. However, this model, though spectacular, has very complex features in term of spatio-temporal evolution, intimately linked with the used parameters. This complexity leads to some difficulties in the definition of the finite element setup in terms of time increment and mesh. Further work will extend the proposed approach to 3D simulations, reactions involving 3 species or more, and mechanical coupling.

To include mechanical DOFs, in the frame of hydrogen transport-trapping-thermomechanic interactions especially, it will be only necessary to introduce in the UELs the related contribution to the weak 
(a) Element size:

$0.01^{2} \mathrm{~mm}^{2}$

(b) Element size:

$0.025^{2} \mathrm{~mm}^{2}$

(c) Element size:

$0.05^{2} \mathrm{~mm}^{2}$
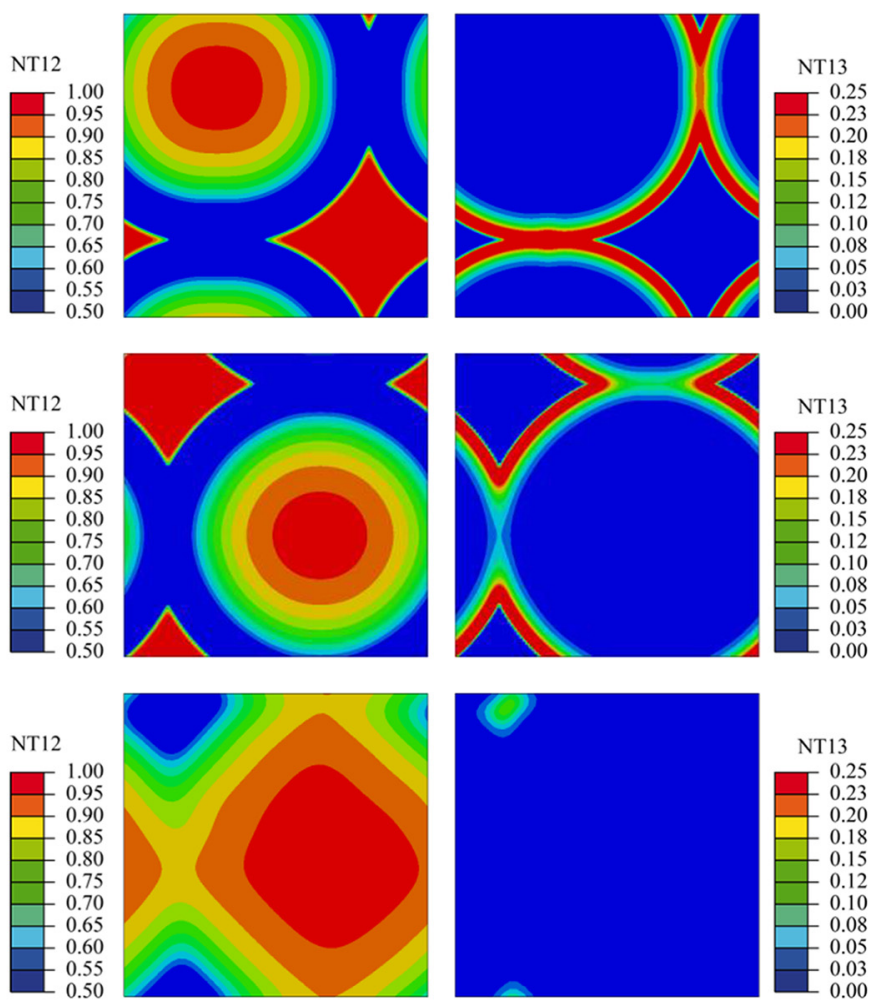

Fig. 9. Influence of the element size on the Abaqus results at $t=800 \mathrm{~s}$ for $u$ (left) and $v$ (right), with $(F, k)=(0.006,0.037)$.

(a) Reference

pattern for $u$ [44]

(a) Element size:

$0.01^{2} \mathrm{~mm}^{2}$, time

increment: $10 \mathrm{~s}$

(b) Element size:

$0.025^{2} \mathrm{~mm}^{2}$, time

increment: $1 \mathrm{~s}$
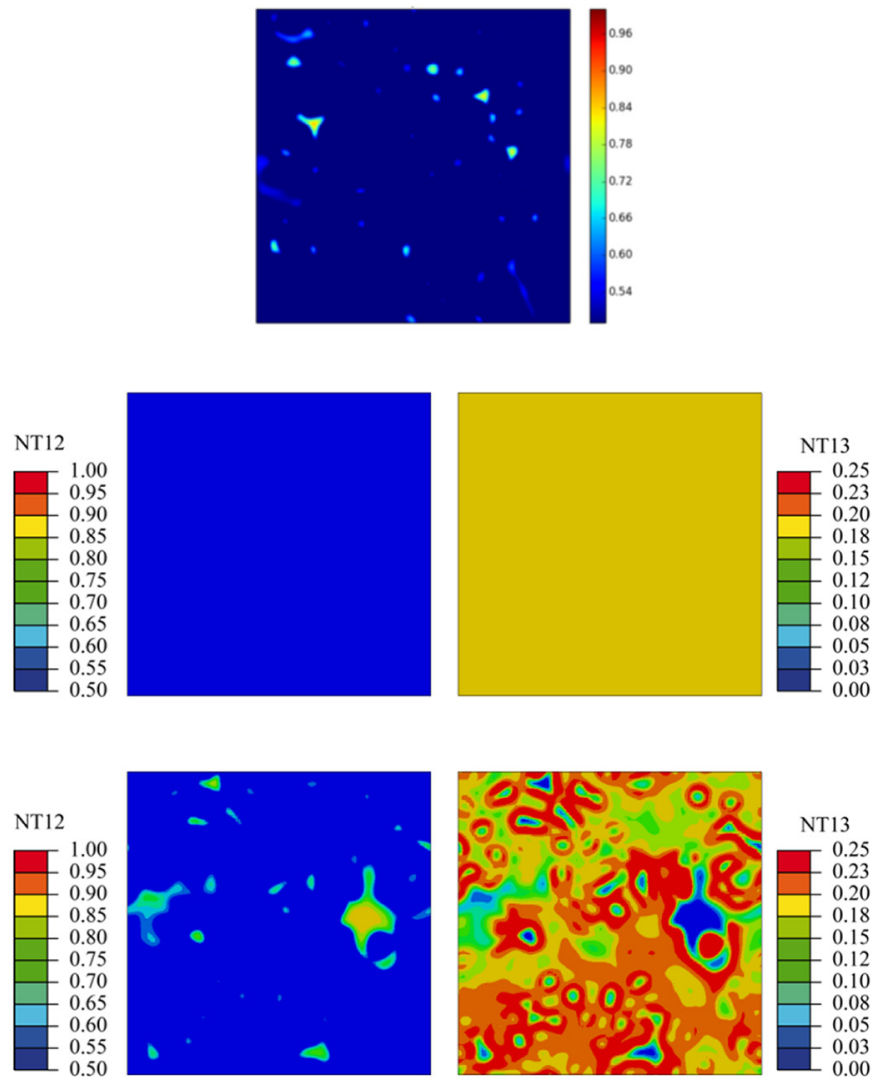

Fig. 10. Influence of the time increment on the Abaqus results at $t=5000 \mathrm{~s}$ for $u$ (left) and $v$ (right), with $(F, k)=(0.022,0.049)$. 


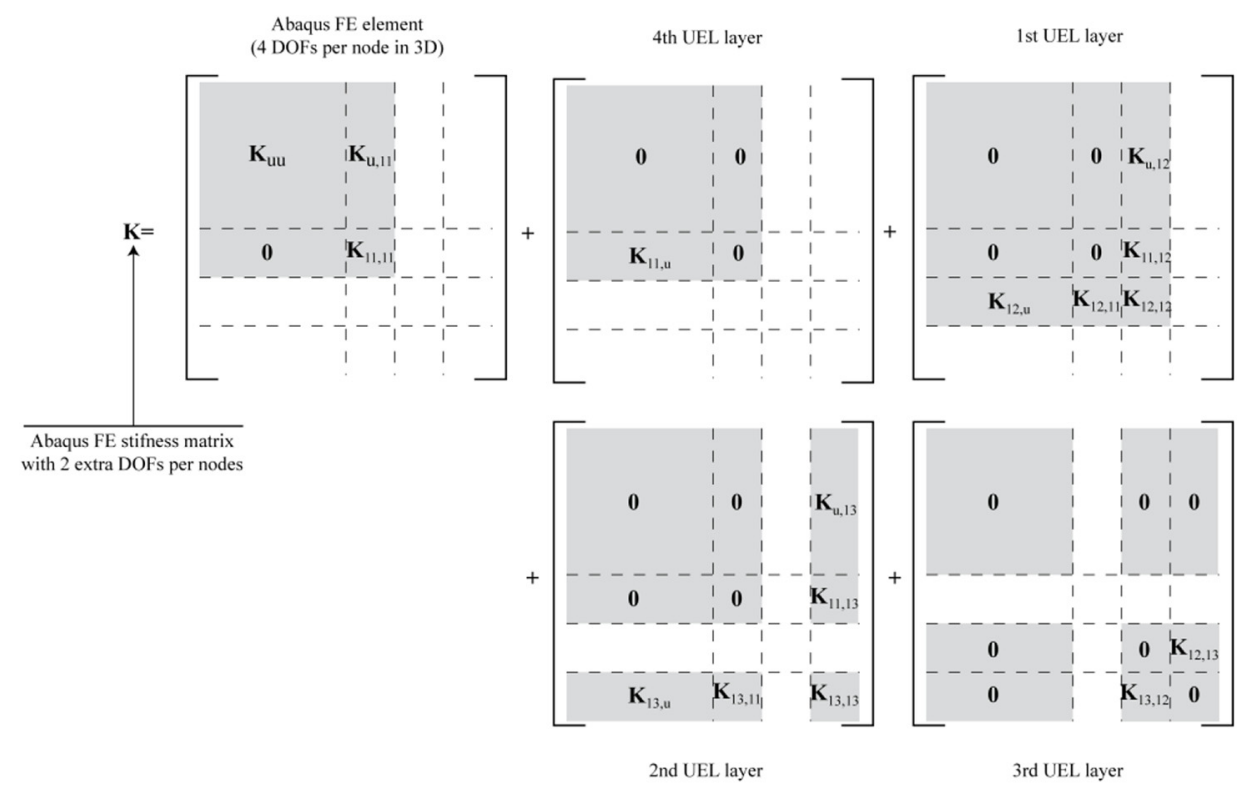

formulation. Furthermore, a 4th layer might be added to include the coupling between DOF 11 and mechanical fields. Equation (1) thus becomes:

$$
\text { see equation (5) above. }
$$

Such an extension work is in progress [51].

\section{Nomenclature}

$\begin{array}{ll}U \text { and } V & \text { Species used in the Gray-Scott equation } \\ F & \text { Feed rate of } U\left(\mathrm{~mol} \mathrm{~s}^{-1}\right) \\ k & \text { Kill rate of } V\left(\mathrm{~mol} \mathrm{~s}^{-1}\right) \\ u_{v} & \text { Diffusion coefficient of } U\left(\mathrm{~m}^{2} \mathrm{~s}^{-1}\right) \\ D_{v} & \text { Diffusion coefficient of } V\left(\mathrm{~m}^{2} \mathrm{~s}^{-1}\right) \\ u & \text { Concentration of } U\left(\mathrm{~mol} \mathrm{~m}^{-3}\right) \\ v & \text { Concentration of } V\left(\mathrm{~mol} \mathrm{~m}^{-3}\right)\end{array}$

The authors would like to thank the financial support of Labex SEAM (Science and Engineering for advanced Materials and Devices), ANR 11 LABX 086, ANR 11 IDEX 05 02, and through the funding of the PANAME-HYSTME (PlasmA-NAnoMEtals interactions: HYdrogen STorage of MEtals) project. This work has been carried out within the framework of the EUROfusion Consortium and has received funding from the Euratom research and training programme 2014-2018 and 2019-2020 under grant agreement No 633053. The views and opinions expressed herein do not necessarily reflect those of the European Commission. This work has been also carried out within the framework of the French Federation for Magnetic Fusion Studies (FR-FCM) under KHMeR2 (Kinetic Hydrogen trapping and Mechanical effects of hydrogen isotopes Retention in metal surfaces) project.

\section{References}

[1] J.P. Hirth, Effects of hydrogen on the properties of iron and steel, Metal. Trans. A 11, 861-890 (1980)
[2] B.E. Sar, S. Fréour, A. Célino, F. Jacquemin, Accounting for differential swelling in the multi-physics modeling of the diffusive behavior of a tubular polymer structure, J. Compos. Mater. 49, 2375-2387 (2014)

[3] K. Derrien, P. Gilormini, Interaction between stress and diffusion in polymers, Defect Diffus. Forum 258-260, 447452 (2006)

[4] A. McNabb, P.K. Foster, A new analysis of the diffusion of hydrogen in iron and ferritic steels, Trans. Metall. Soc. AIME 227, 618-627 (1963)

[5] H.G. Carter, K.G. Kibler, Langmuir-type model for anomalous moisture diffusion in composite resins, J. Compos. Mater. 12, 118-131 (1978)

[6] P. Sofronis, R.M. McMeeking, Numerical analysis of hydrogen transport near a blunting crack tip, J. Mech. Phys. Solids 37, 317-350 (1989)

[7] I.M. Bernstein, The role of hydrogen in the embrittlement of iron and steel, Mater. Sci. Eng. A 6, 1-19 (1970)

[8] C.-H. Shen, G.S. Springer, Effects of moisture and temperature on the tensile strength of composite materials, J. Compos. Mater. 11, 2-16 (1977)

[9] A.H.M. Krom, R.W.J. Koers, A.D. Bakker, Hydrogen transport near a blunting crack tip, J. Mech. Phys. Solids 47, 971-992 (1999)

[10] C.-S. Oh, Y.J. Kim, K.B. Yoon, Coupled analysis of hydrogen transport using ABAQUS, J. Solid Mech. Mater. Eng. 4, 908-917 (2010)

[11] N. Yazdipour, A.J. Haq, K. Muzaka, E.V. Pereloma, 2D modelling of the effect of grain size on hydrogen diffusion in X70 steel, Comput. Mater. Sci. 56, 49-57 (2012)

[12] T. Peret, A. Clement, S. Fréour, F. Jacquemin, Numerical transient hygro-elastic analyses of reinforced Fickian and non-Fickian polymers, Compos. Struct. 116, 395-403 (2014)

[13] Y. Charles, T.H. Nguyen, M. Gaspérini, Comparison of hydrogen transport through pre-deformed synthetic polycrystals and homogeneous samples by finite element analysis, Int. J. Hydrogen Energy 42, 20336-20350 (2017)

[14] Y. Charles, T.H. Nguyen, M. Gaspérini, FE simulation of the influence of plastic strain on hydrogen distribution during an U-bend test, Int. J. Mech. Sci. 120, 214-224 (2017) 
[15] S. Benannoune, Y. Charles, J. Mougenot, M. Gaspérini, Numerical simulation of the transient hydrogen trapping process using an analytical approximation of the McNabb and Foster equation, Int. J. Hydrogen Energy 43, 9083-9093 (2018)

[16] Y. Zhao, P. Stein, Y. Bai, M. Al-Siraj, Y. Yang, B.-X. Xu, A review on modeling of electro-chemo-mechanics in lithiumion batteries, J. Power Sources 413, 259-283 (2019)

[17] D. Kuhl, F. Bangert, G. Meschke, Coupled chemo-mechanical deterioration of cementitious materials. Part II: Numerical methods and simulations, Int. J. Solids Struct. 41, 41-67 (2004)

[18] T. Poulet, A. Karrech, K. Regenauer-Lieb, L. Fisher, P. Schaubs, Thermal-hydraulic-mechanical-chemical coupling with damage mechanics using ESCRIPTRT and ABAQUS, Tectonophysics 526-529, 124-132 (2012)

[19] A. Karrech, Non-equilibrium thermodynamics for fully coupled thermal hydraulic mechanical chemical processes, J. Mech. Phys. Solids 61, 819-837 (2013)

[20] S. Benannoune, Y. Charles, J. Mougenot, M. Gaspérini, G. De Temmerman, Numerical simulation by finite element modelling of diffusion and transient hydrogen trapping processes in plasma facing components, Nucl. Mater. Energy 19, 42-46 (2019)

[21] P. Gray, S.K. Scott, Autocatalytic reactions in the isothermal, continuous stirred tank reactor: isolas and other forms of multistability, Chem. Eng. Sci. 38, 29-43 (1983)

[22] P. Gray, S.K. Scott, Autocatalytic reactions in the isothermal, continuous stirred tank reactor: oscillations and instabilities in the system $\mathrm{A}+2 \mathrm{~B} \rightarrow 3 \mathrm{~B}$; $\mathrm{B} \rightarrow \mathrm{C}$, Chem. Eng. Sci. 39, 1087-1097 (1984)

[23] G. Molnár, A. Gravouil, 2D and 3D Abaqus implementation of a robust staggered phase-field solution for modeling brittle fracture, Finite Elem. Anal. Des. 130, 27-38 (2017)

[24] E. Martínez-Pañeda, A. Golahmar, C.F. Niordson, A phase field formulation for hydrogen assisted cracking, Comput. Methods Appl. Mech. Eng. 342, 742-761 (2018)

[25] Simulia, Abaqus User subroutines reference guide, Dassault Système (2011)

[26] S.A. Chester, C.V. Di Leo, L. Anand, A finite element implementation of a coupled diffusion-deformation theory for elastomeric gels, Int. J. Solids Struct. 52, 1-18 (2015)

[27] J. Chen, H. Wang, P. Yu, S. Shen, A finite element implementation of a fully coupled mechanical-chemical theory, Int. J. Appl. Mech. 09, 1750040 (2017)

[28] Simulia, Abaqus Analysis User's Manual, Dassault System (2011)

[29] Y. Charles, A finite element formulation to model extrinsic interfacial behavior, Finite Elem. Anal. Des. 88, 55-66 (2014)

[30] R.A. Barrio, Turing systems: a general model for complex patterns in nature, in: I. Licata, A. Sakaji (Eds.), Physics of Emergence and Organization, World Scientific (2008) 267-296

[31] H. Meinhardt, Wie Schnecken sich in Schale werfen, Springer Berlin Heidelberg, Berlin, Heidelberg, 1997

[32] B. Rudovics, E. Barillot, P.W. Davies, E. Dulos, J. Boissonade, P. De Kepper, Experimental studies and quantitative modeling of turing patterns in the (chlorine dioxide, iodine, malonic acid) reaction, J. Phys. Chem. A 103, 1790-1800 (1999)
[33] I. Szalai, P. De Kepper, Pattern formation in the ferrocyanide-iodate-sulfite reaction: the control of space scale separation, Chaos 18, 026105 (2008)

[34] P.W. Davies, P. Blanchedeau, A.E. Dulos, P. De Kepper, Dividing blobs, chemical flowers, and patterned islands in a reaction-diffusion system, Am. Chem. Soc. (1998). https:// doi.org/10.1021/jp982034n.

[35] A.S. Mikhailov, G. Ertl, The Belousov-Zhabotinsky Reaction, in: Chemical Complexity, Springer, Cham, Cham (2017) 89-103

[36] J.E. Pearson, Complex Patterns in a Simple System, Science 261, 189-192 (1993)

[37] W. Mazin, K.E. Rasmussen, E. Mosekilde, P. Borckmans, G. Dewel, Pattern formation in the bistable Gray-Scott model, Math. Comput. Simul. 40, 371-396 (1996)

[38] F. Lesmes, D. Hochberg, F. Morán, J. Pérez-Mercader, Noise-controlled self-replicating patterns, Phys. Rev. Lett. 91, $238301(2003)$

[39] H. Wang, Z. Fu, X. Xu, Q. Ouyang, Pattern formation induced by internal microscopic fluctuations, J. Phys. Chem. A 111, 1265-1270 (2007)

[40] W. Wang, Y. Lin, F. Yang, L. Zhang, Y. Tan, Numerical study of pattern formation in an extended Gray-Scott model, Commun. Nonlinear Sci. Numer. Simul. 16, 2016-2026 (2011)

[41] R.P. Munafo, Stable localized moving patterns in the 2-D Gray-Scott model. https://arxiv.org/abs/1501.01990 (2014)

[42] A. Adamatzky, Generative complexity of Gray-Scott model, Commun. Nonlinear Sci. Numer. Simul. 56, 457-466 (2018)

[43] T. Hutton, R.P. Munafo, A. Trevorrow, T. Rokicki, D. Wills, Ready, a cross-platform implementation of various reactiondiffusion systems, (2011) GNU General Public License. https://github.com/GollyGang/ready/

[44] D. Bennewies, Final Report, Waterloo, Ontario, Canada, 2012. https://github.com/derekrb/gray-scott

[45] F. Buric, Pattern formation and chemical evolution in extended Gray-Scott models, Chalmers University of Technology, Göteborg, Sweden, 2014. http://github.com/phil-b-mt/egs

[46] R.P. Munafo, Reaction-Diffusion by the Gray-Scott Model: Pearson's Parametrization. https://mrob.com/pub/comp/ xmorphia/ (accessed April 2017)

[47] D. Gizaw, Finite element method applied to gray-scott reaction-diffusion problem. Using FEniCs Software, Adv. Phys. Theories Appl. 58, 5-8 (2016)

[48] T. Lun, Finite Element Simulation of Pattern Formation in Gray-Scott Model, Researchgate.Net. (2016). https://doi. org $/ 10.13140 /$ RG.2.1.2780.6329

[49] C. Xie, X. Hu, Finite element simulations with adaptively moving mesh for the reaction diffusion system, Numer. Math. Theory Methods Appl. 9, 686-704 (2016)

[50] T. Lun, Gray-Scott Coupled Mechanical Model, Researchgate.Net. (2016). https://doi.org/10.13140/RG.2.1.3994. 3928

[51] S. Benannoune, Y. Charles, J. Mougenot, M. Gaspérini, G. De Temmerman, Multidimensional finite element simulations of the diffusion and trapping of hydrogen in plasmafacing components including thermal expansion, Phys. Scripta (2020)

Cite this article as: E. Vasikaran, Y. Charles, P. Gilormini, Implementation of a reaction-diffusion process in the Abaqus finite element software, Mechanics \& Industry 21, 508 (2020) 